\title{
Stem cells and cell-based therapies for cerebral palsy: a call for rigor
}

\author{
Lauren L. Jantzie ${ }^{1}$, Joseph Scafidi ${ }^{2}$ and Shenandoah Robinson ${ }^{3}$
}

Cell-based therapies hold significant promise for infants at risk for cerebral palsy (CP) from perinatal brain injury (PBI). PBI leading to CP results from multifaceted damage to neural cells. Complex developing neural networks are injured by neural cell damage plus unique perturbations in cell signaling. Given that cell-based therapies can simultaneously repair multiple injured neural components during critical neurodevelopmental windows, these interventions potentially offer efficacy for patients with CP. Currently, the use of cell-based interventions in infants at risk for CP is limited by critical gaps in knowledge. In this review, we will highlight key questions facing the field, including: Who are optimal candidates for treatment? What are the goals of therapeutic interventions? What are the best strategies for agent delivery, including timing, dosage, location, and type? And, how are short- and long-term efficacy reliably tracked? Challenges unique to treating PBI with cell-based therapies, and lessons learned from cell-based therapies in closely related neurological disorders in the mature central nervous system, will be reviewed. Our goal is to update pediatric specialists who may be counseling families about the current state of the field. Finally, we will evaluate how rigor can be increased in the field to ensure the safety and best interests of this vulnerable patient population.

C erebral palsy (CP), the most common physical disability in childhood, describes a group of permanent disorders that affect movement, muscle tone, and/or posture, and results from non-progressive disturbances of the developing central nervous system (CNS) (1). The prevalence of CP in countries with advanced medical care is $2.22-2.9$ per 1,000 $(2-4)$, and is likely higher in economically disadvantaged locations. Motor deficits vary among individuals. To facilitate classification, patients are grouped into five levels of severity using the Gross Motor Function Classification System (5-7). Although the overall prevalence of $\mathrm{CP}$ has been stable over time, patterns of motor deficits have shifted. Currently, fewer children born at term are diagnosed with $\mathrm{CP}$, while more preterm survivors have CP (8). A pattern shift was observed in the United States from 2006 to 2010, with an increase in the mildest and most severe CP (Gross Motor Function Classification System I $(38 \rightarrow 48 \%)$ and V $(17 \rightarrow 20 \%)$, with a reduction in II $(16 \rightarrow 8 \%)$ and III $(13 \rightarrow 9 \%)$ (IV was stable)) (3). These shifts over relatively short time intervals emphasize the need for clinical trial designs with concurrent controls. Most individuals with $\mathrm{CP}$ experience additional neurological comorbidities, related to speech, cognition, behavior, epilepsy, and pain that affect them throughout their lifespan. For example, among people with CP, $75 \%$ suffer pain, $50 \%$ have intellectual disability, 33\% cannot walk, and 25\% cannot talk, $25 \%$ have epilepsy, $25 \%$ have incontinence, and $10 \%$ are blind (9). Both motor impairment and non-motor comorbidities vary across individuals, emphasizing that outcome metrics need to assess multiple domains (10). Given the confounding factors of individual deficit patterns from comorbidities, and regional and ethnic/genetic differences in motor deficits, emerging interventions will likely need to be tested in specific populations of patients at risk for, or diagnosed with CP from specific etiologies.

Etiological factors associated with $\mathrm{CP}$ include chorioamnionitis and preterm birth, maternal and/or neonatal infections, intrauterine growth restriction, neonatal encephalopathy, infantile traumatic brain injury, and genetic mutations (11-13). Congenital abnormalities in children with $\mathrm{CP}$ are more common than appreciated previously (13). Importantly, many children with $\mathrm{CP}$ have either multiple overlapping etiologies $(14,15)$ or fail to have a specific cause identified; both factors impact clinical trial design and outcomes (16). Numerous rehabilitative, medical, and surgical interventions help people with CP maximize functional skills (17). Unlike many other neurological conditions where scores have been standardized to compare outcomes for clinical trials, however, rigorous standardized assessments of multidisciplinary outcomes for CP interventions are rare (16). No treatment currently exists that cures CP. In sum, the lack of curative interventions has encouraged caregivers to seek promising therapies that may lack scientific and clinical evidence of effectiveness.

\footnotetext{
${ }^{1}$ Departments of Pediatrics and Neurosciences, University of New Mexico School of Medicine, Albuquerque, New Mexico; ${ }^{2}$ Department of Neurology, Children's National Health System, Washington, DC; ${ }^{3}$ Division of Pediatric Neurosurgery, Johns Hopkins School of Medicine, Johns Hopkins University, Baltimore, Maryland. Correspondence: Shenandoah Robinson (srobin81@jhmi.edu)

Received 9 June 2017; accepted 22 July 2017; advance online publication 1 November 2017. doi:10.1038/pr.2017.233
} 


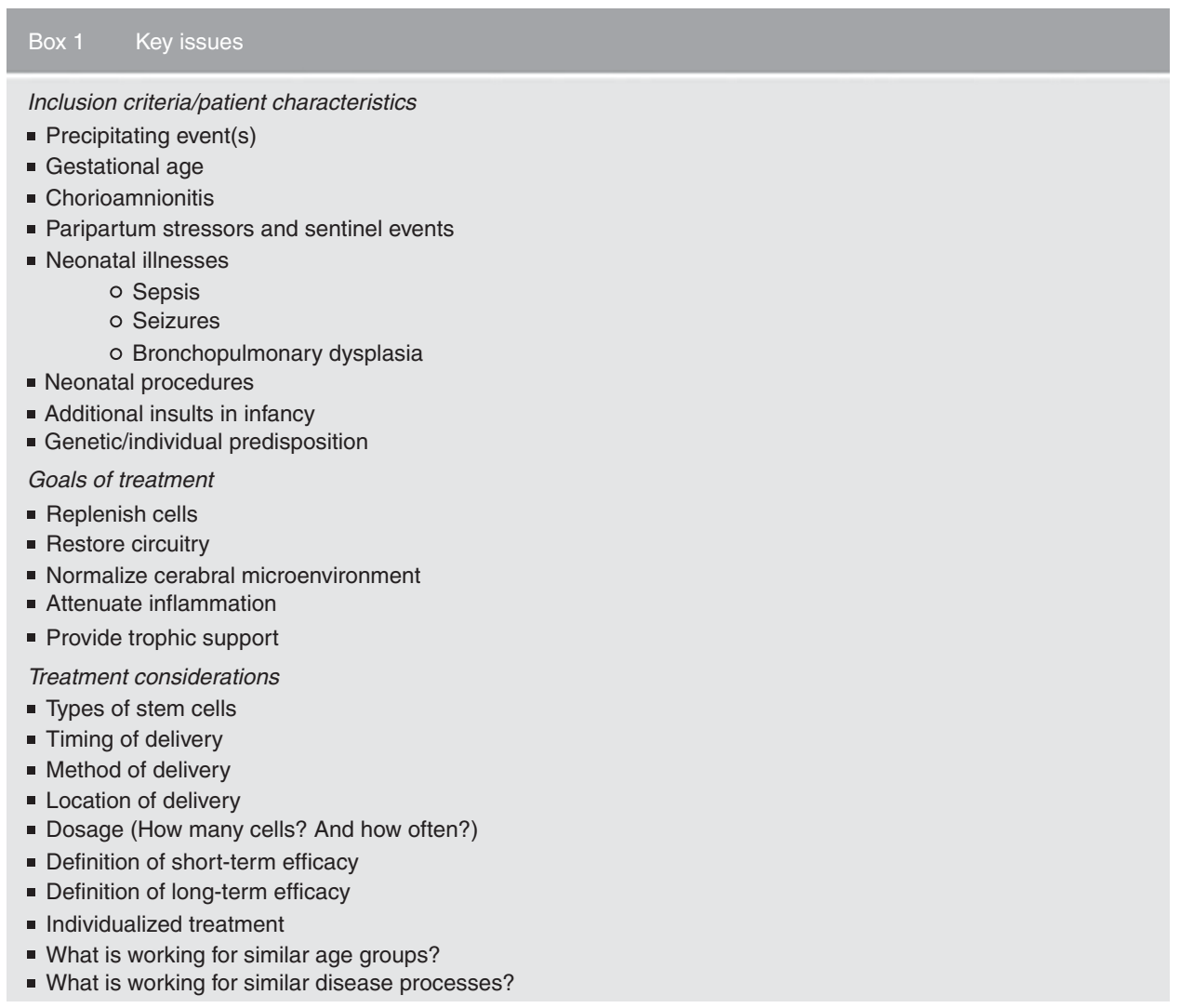

Figure 1. Key issues regarding stem cell use for cerebral palsy.

Recently, cell-based therapies have been identified as a high-priority research topic for CP. Cell-based therapies might be particularly effective for $\mathrm{CP}$ because the CNS damage is non-progressive, and typically involves multiple neural cell types plus CNS inflammation. Both processes are common to other neurological disorders that are already pioneering stem cell research (18-20). The literature pertaining to cell-based therapies has grown considerably over the past 5 years, but the key questions remain (Figure 1). Specialists who treat patients with $\mathrm{CP}$ have been consistently and appropriately forthright with their concerns (21-26). While cell-based therapies represent new strategies for neural repair, much remains to be learned (27-29). Guidelines for clinical translation of stem cells were released to promote rigorous scientific inquiry and careful ethical deliberations regarding stem cell science (30). Moving forward, reviewers and editors should require compliance with these guidelines. Given the expansive literature on these topics and guidelines for clinical translation (30), several themes and ethical issues related to cell-based therapies have recently been addressed well by others (31). Similarly, powerfully framed discussions of stem cells in the media, and marginalization of vulnerable patients and families in need of hope and access to treatment are reviewed eloquently elsewhere (32). Here, we focus on key scientific aspects of cell-based therapies pertinent to $\mathrm{CP}$, and their translation to early clinical trials.
To move cell-based interventions for the treatment of $\mathrm{CP}$ forward, rigorous evaluation of preclinical science is paramount. Developmental programs in the perinatal CNS are intricate, with integrated spatiotemporal cascades that are only partially understood. Preclinical emphasis on the complex dynamic between environmental perturbations, such as ischemia, inflammation, and infection, and their cumulative impact on CNS and musculoskeletal system development are required. Because of the varied etiologies of CP $(14,15)$, and our emerging knowledge of complex neuronal-glial interactions after injury (18), preclinical models that accurately replicate etiologies of $\mathrm{CP}$, the chronic deficits observed in $\mathrm{CP}$, and administration in the chronic phase of $\mathrm{CP}$ are paramount. As an example of the rigorous step-wise approach needed, human CNS-derived stem cell lines that successfully repaired thoracic spinal cord injury in rodent models failed to produce improvement with cervical spinal cord injury, the most pressing human form of spinal cord injury that was targeted for early clinical trials (33). This work emphasizes the importance of testing specific agents destined for human trials in clinically relevant and accurate preclinical models (33). Preclinical models that accurately recapitulate CP in each of its etiologies and manifestations are essential, particularly those reflecting chronic motor deficits (34-37). Moreover, preclinical models that encompass placental contributions and inflammation may be of high utility for CNS injury from chorioamnionitis and preterm birth $(11,37,38)$, the leading 
cause of CP in the United States. Multifactorial etiologies will be more challenging to model well in animals.

Similarly, clinical outcomes in trials should include specific, reliable a priori measures of efficacy that are also used to evaluate current medical and surgical interventions. Outcomes should include the full spectrum of motor impairment in CP, including measures of spasticity and dystonia (10). Specifically, a Common Data Elements approach, such as that used in pediatric traumatic brain injury (39), is needed to standardize outcomes and analyses across trials. To guide human trials, robust tracking of acute and chronic outcomes using age-appropriate, validated measures, as well as risks and benefits are needed. High-resolution imaging of both structural and functional CNS connectivity is essential (40). In addition to childhood imaging biomarkers, neonatal neuroimaging, electrocorticography, and systemic physiologic serum biomarkers are warranted to provide subject stratification and timely feedback for clinical trial design. More sensitive functional outcomes are needed to delineate an individual's natural history from real therapeutic benefits of cell-based therapy. Specific, molecular-targeted biomarkers are needed to capture changes in neural cell development and injury to allow individually tailored application of interventions, similar to the application of precision medicine in cancer treatment.

\section{WHO ARE THE OPTIMAL CANDIDATES FOR TREATMENT?}

The etiology of $\mathrm{CP}$ is heterogeneous, and some children have $\mathrm{CP}$ from mixed or unknown etiologies. To identify who would be best served by a specific cell-based therapy, the etiology and extent of CP for those screened for clinical trials should be clarified, ideally by a combination of clinical history, functional measures, serum biomarkers, and structural and functional imaging. As the etiology of CP largely predicts clinical outcome and therapeutic response, what cell-based therapies can accomplish will be partially defined by the local CNS microenvironment after injury. Notably, etiology may also have bearing on immune response and persistence of an inflammatory reaction that may dictate candidates for therapy (13). Preterm birth and in utero exposure to infection and inflammation are known precipitators of $\mathrm{CP}$ and sustained systemic prenatal and postnatal inflammation $(11,14,41,42)$. Chorioamnionitis and fetal inflammatory response syndrome (FIRS) are highly associated with the subsequent diagnosis of CP (43-45). Neonatal illnesses including sepsis, epilepsy, and bronchopulmonary dysplasia likely contribute to the functional manifestation of deficits in $\mathrm{CP}$ and the therapeutic response. To identify predictors of severe motor impairment at 5 years of age, 1,469 former preemies $(<1,250 \mathrm{~g}$ at birth) from the United States, Europe, Israel, and Australia were evaluated (46). Almost $20 \%$ had severe motor impairment that could be predicted well by seven neonatal, six term equivalent, and seven infancy variables (46). If a specific cellbased therapy is to be tested in an early clinical trial, a validated scoring system to predict eligibility and stratification is essential. Ideally, stratification would include biomarkers with body fluids and/or imaging/electrocorticography to account for an individual's genetic predisposition and his or her likelihood to respond to treatment. This process would be similar to how cancer treatment has evolved from basic descriptors of histopathology to using molecular and genetic signatures to individualize and tailor therapeutic regimens. Stratification of older infants, toddlers, and children will likely be even more challenging as the perinatal data will be remote and harder to quantify with biomarkers. Imaging biomarkers may prove particularly useful for toddlers and children to improve stratification for early clinical trials.

\section{WHAT ARE THE GOALS OF THERAPEUTIC INTERVENTIONS?}

While the primary goals of cell-based therapies is to replenish cells, restore circuitry, normalize the microenvironment, and thus CNS activity, accomplishing this depends on a spectrum of diverse neural cells interacting in concert in the correct spatiotemporal pattern (47). Unlike neurological diseases with specific neuronal sub-populations presumed to be the primary pathogenic targets (i.e., nigrostriatal dopaminergic neurons in Parkinson's disease), most etiologies of CP encompass widespread glial activation and injury, plus loss of multiple neuronal subtypes, including migrating GABAergic interneurons, excitatory neurons, and subplate neurons (48-50). Moreover, multiple types of neural cells are involved in the pathophysiology of CP (51). In this context, some neural cell populations are likely more resistant to injury than others, and by contrast, some cell types are likely more easily replenished than others. For example, pyramidal neurons dictate the position of cortical interneurons through modulation of local neuronal activity and soluble factors in the local microenvironment (52). Replicating such interactions during repair to guide new interneurons will be challenging. Neurons in particular are heavily influenced by adjacent cells including astrocytes, oligodendrocytes, and microglia, and the local extracellular microenvironment. Like neuronal subtypes, multiple subtypes of astrocytes, oligodendrocytes, microglia exist based on morphological, regional, and functional phenotypes $(53,54)$. While morphological classifications and changes are perhaps the most obvious, glial populations vary by brain region and age, each with their own unique developmental trajectories $(54,55)$. Further, astrocytes and microglia are driving forces of the CNS neuroinflammatory response that shapes neuronal and oligodendroglial survival and maturation (56). Thus, the glial inflammatory responses strongly reflect individual patient characteristics and genetic predisposition to both injury and recovery.

The glial neuroinflammatory response is closely intertwined with the local microenvironment with the extracellular matrix, immune cells, cytokines, and chemokines. Injury during development also affects neurotransmitters, receptor subtypes, glutamatergic and GABAergic neurotransmission, and levels of cotransporters (48,57-59). In a study by Lin et al. (60), white blood cells from 5-year-old children who were born extremely preterm were collected and challenged with lipopolysaccharide, a bacterial endotoxin used to stimulate 


\section{Review | Jantzie et al.}

inflammatory responses. Leukocytes from preterm survivors with $\mathrm{CP}$ had grossly elevated inflammatory responses characterized by high tumor necrosis factor levels, while preterm survivors without $\mathrm{CP}$ did not (60). Even at an unstimulated baseline, the former preemies with $\mathrm{CP}$ had much higher levels of inflammatory molecules (60). This study highlights the ongoing role of the immune system in CP pathophysiology, and also emphasizes that immune function may be different in patients with CP. These data also emphasize that clinical biomarkers are needed to assess the state of the immature and mature immune system, systemic, and neuroinflammatory responses in $\mathrm{CP}$ patients. This is required to answer the question as to whether those who are predisposed to, or have $\mathrm{CP}$, will respond differently to cellbased therapies in the context of the diverse trajectory of all neural cell populations.

\section{TYPES OF STEM CELLS AND CELL-BASED THERAPIES}

The distinguishable phenotypic features of stems cells are an integral component of the understanding the biology and potential for cell-based therapies in CP (61-64). Indeed, to determine their safety, efficacy, and putative mechanisms of beneficial action, stem cell identity is paramount. It is necessary to determine the source of stem cells and thus mechanisms of action associated with cell therapy. Notably, diverse types of stem cells have been identified for potential use in CP (Figure 2). Here we focus on those cells most commonly proposed for CP. While autologous cells may superficially seem appealing, allogenic cells are likely better for preterm neonates because of limited quantities, stem cells arising in the context of the etiology of $\mathrm{CP}$ (e.g., fetal inflammatory response syndrome), and immune immaturity (43). Both autologous and allogenic stem cells can be challenging to reliably produce for clinical trials (65-67).

Umbilical cord blood (UCB) is a rich source of mononuclear cells that contains high levels of primitive multipotent stem/progenitors, and UCB contains mesenchymal stem cells (MSCs) and a large number of endothelial cell precursors $(68,69)$. Cells from UCB are immune naïve and are also capable of differentiating into other cell phenotypes, including neural lineages $(70,71)$. Despite being relatively easy to obtain, feasible, and potentially efficacious for use in neonatal brain injury, the quantity of umbilical cord blood mononuclear cells obtained from each infant and the amount of stem cells within each batch are variable and heterogeneous. Similarly, preterm babies may not be the best candidates for autologous stem cell transplants as collection volumes are proportional to gestational age, with younger infants yielding the smallest samples; it is unknown how maternal and fetal complications including placental insufficiency, intrauterine growth restriction, and preeclampsia alter the relative proportion of stem and immune cells (72-74). Naturally, this brings concerns about standardization of optimal cell preparation, function, and dosing, which preclinical studies indicate are predictive of efficacy $(22,75)$.

Individual MSCs are readily isolated from placenta, Wharton's jelly, and umbilical cord, as well as adult bone marrow and adipose tissue (76). Regardless of the cell source, each MSC population exhibits a distinguishing and specific set of cell-surface antigens that define multipotency. Typically, MSCs are well tolerated immunologically given their low expression of major histocompatibility complex I and lack of major histocompatibility complex II molecules (77). Wharton's jelly-derived MSCs are less differentiated than bone marrow-derived MSCs, and thus may be more likely to produce neural cells. Similarly, cells derived from Wharton's jelly or UCB show lower immunogenicity, higher proliferative capacity, and increased paracrine potency compared with adult tissue-derived MSCs $(78,79)$.

All types of MSCs can be easily expanded in culture and maintain their undifferentiated state. This is an important consideration when sources of MSCs are an issue and may be

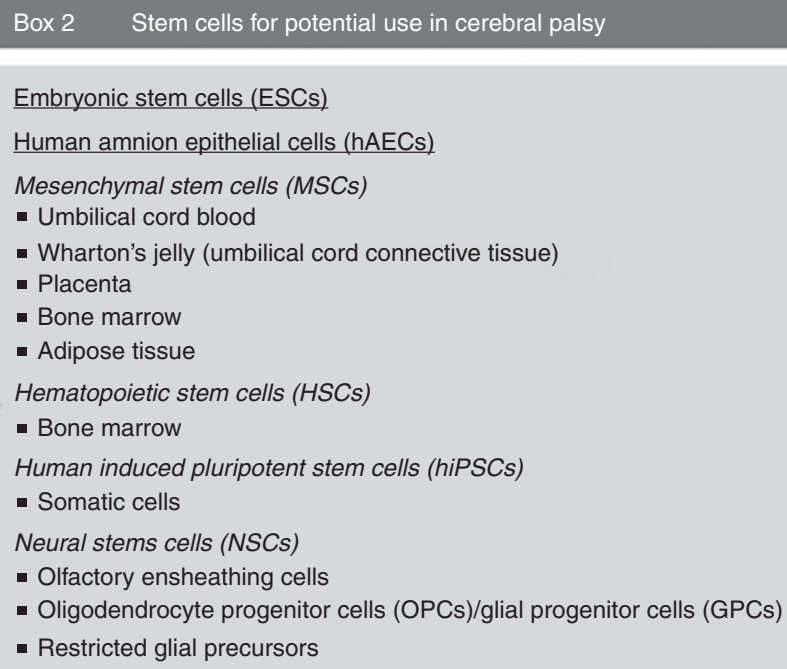

Figure 2. Type of stem cells for potential use in cerebral palsy. 


\section{Cell-based therapies for cerebral palsy Review}

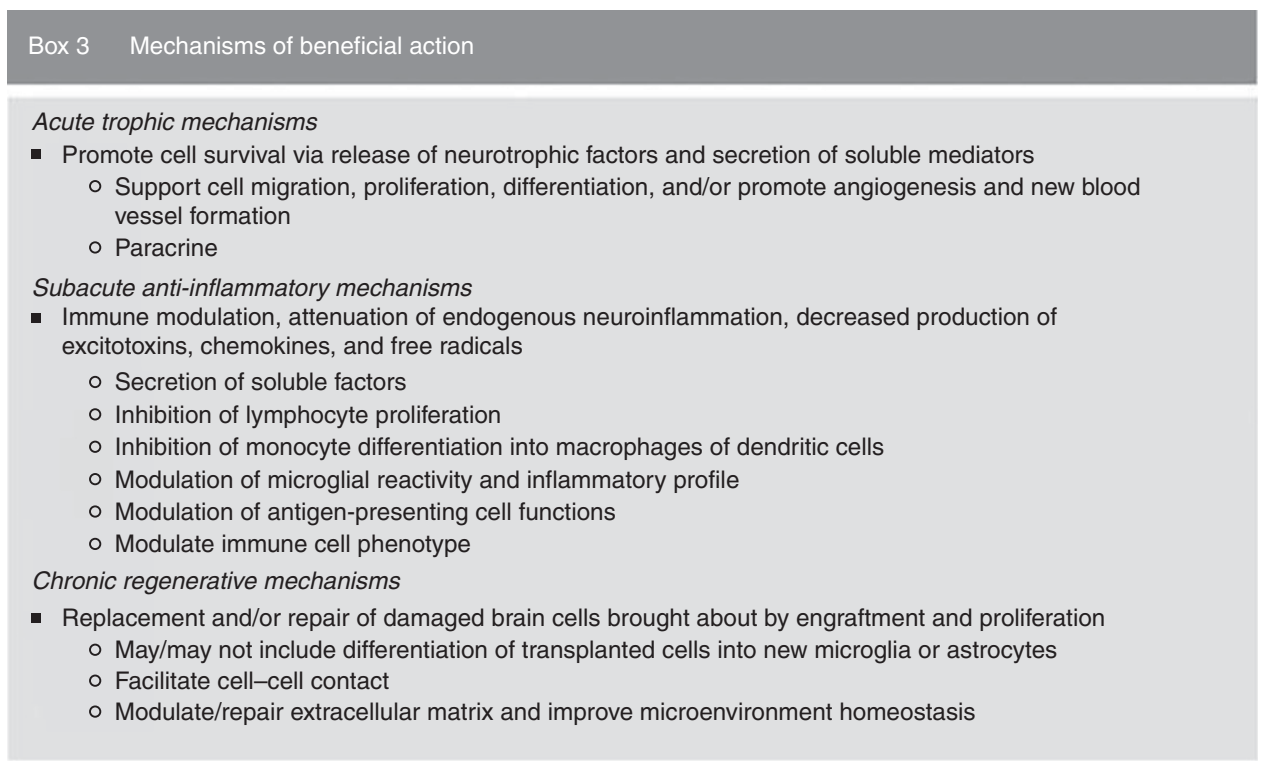

Figure 3. Putative beneficial mechanisms of action of stem cells for use in cerebral palsy.

necessary to provide a therapeutic dose. While autologous stem cells from UCB can be an advantage because of their ability to bypass host immune cell response and graft rejection (80), there are limitations. Most children with CP do not have bio-banked UCB. In this context, several preclinical and clinical trials have shown that allogeneic UCB stem cells have therapeutic potential for patients with CP $(81,82)$. Full understanding, however, of their safety and efficacy is still limited. One clinical trial for $\mathrm{CP}$ has assessed the putative difference between autologous and allogeneic stem cells (83). Treatment with allogeneic UCB stem cells significantly decreased numerous proinflammatory cytokines (interleukin- 6 , interleukin- $1 \alpha$, tumor necrosis factor- $\beta$, and RANTES (regulated on activation, normal $\mathrm{T}$ cell expressed and secreted)), and showed improvement in motor and social behavior compared with autologous UCB treatment groups (83). More rigorously designed clinical trials are needed to test both safety and efficacy.

Human-induced pluripotent stem cells (hiPSCs) are being explored for numerous neurological disorders that affect the mature CNS. hiPSCs hold the most promise for disorders where a specific defect, such as an enzyme abnormality, has been identified, and importantly, the several weeks needed to generate the cells will not impact outcome $(20,84,85)$. Given the diverse etiologies and perinatal events associated with $\mathrm{CP}$, hiPSCs perhaps hold less promise for some patients with $\mathrm{CP}$ because only a specific cell type is generated after several weeks.

\section{MECHANISMS OF BENEFICIAL EFFECT}

Stem cells are multipotent and respond to microenvironmental cues to guide differentiation and cellular activity (86). Although the mechanisms are incompletely understood, transplanted stem cells have proven to be capable of replacing neurons, glia, and vascular cells, and releasing trophic factors.
The primary mode of action involved in stem cell-mediated functional recovery with in $\mathrm{CP}$ is derived from paracrine effects, and less so from replacement or engraftment (Figure 3) $(21,26,87,88)$. Secreted factors facilitate neurogenesis, angiogenesis, synaptogenesis, and immunomodulation (89). Notably, effects of stem cells may differ based on cell origin and the time from administration. Early after administration, restoration of the microenvironment is the mechanism likely driving repair, with notable effects on immune modulation, cell adhesion molecules, including cadherins, selectins, and integrins, and intracellular metabolism (90). In the subacute phase, or weeks after administration, paracrine mechanisms are likely most prominent. Stem cell release of growth factors and other neuromodulators may direct microglial phenotypes, including fine-tuning the local microenvironment to favor anti-inflammatory glial phenotypes (89). Lastly, chronic effects of stem cell therapy may impact neural cell survival, and thus engraftment and formation of direct intracellular interactions to provide maintenance of glial support, neuronal circuits, and myelination of axons (89). Investigators have tested identical cell dosages in a specific injury model, and noted that cells delivered 3 days after the injury were most effective, while cells delivered days earlier or later were not (91). Given that many sick neonates suffer a series of potentially injurious perinatal events, the timing of cell-based treatment, and standardization of dosing regimens during clinical trials becomes even more challenging.

Stem cells also secrete exosomes and other microvesicles designated for precise receptor-mediated intracellular communication. Exosomes carry cargo, including microRNA, proteins, biologically reactive lipids, and receptors that may be beneficial to the injured developing brain. Recently, extracellular vesicles from mesenchymal stromal cells have been shown to rescue cognitive impairments after traumatic brain 


\section{Review | Jantzie et al.}

injury (92). This investigation is one of several publications showing the benefits of exosomes in repair. Similarly, Drommelschmidt et al. (93) demonstrated administration of human MSC-derived exosomes after early postnatal inflammatory injury in rats improved in short- and long-term outcomes, including cognition, motor skills, and microstructural diffusion tensor imaging abnormalities (93). Indeed, exosomes hold significant promise as they may be as efficacious and relatively easier to consistently produce and administer for clinical trials.

Most restoration of function in $\mathrm{CP}$ after cell-based interventions is likely due to paracrine effects on the CNS microenvironment, rather than direct cell replacement $(88,94)$. Each stem cell type within the context of its environment expresses a specific set of factors, or "secretome" (95-97). Indeed, the secretome potentially offers a more standardized way to quantify and characterize a cell preparation from a specific source, and even to manipulate it in vitro to optimize secreted factors (98). In this way, the cell types and even "dosage strength" could be quantified to allow comparison of interventions across patients and trials, especially from autologous sources.

\section{OPTIMAL LOCATION, DOSAGE, AND TIMING FOR CELL- BASED DELIVERY}

Determining the optimal route of stem cell administration is a key issue to be resolved to advance successful clinical translation for the treatment of CP. Currently, numerous routes of administration have been tested including intraventricular, intrathecal, intranasal, intramuscular, intra-arterial, or intravenous $(88,91,99,100)$. Systemic routes may be adequate for modulating inflammation, but are likely suboptimal for treating local CNS lesions because injected cells may be retained in other organs, and by the inability of cells to cross the blood-brain barrier (99). Similarly, intrathecally injected cells may not diffuse to the brain. Direct cerebral injection may be disadvantageous in young, unstable, and medically fragile infants. In the clinical setting where intraventricular devices are required for cerebrospinal fluid diversion or removal, local administration may be more feasible. Injection location within the brain even may be important. Piao et al. (101) administered human embryonic stem cell-derived oligodendrocyte progenitors after cranial radiation. Cells injected into the cerebellum improved motor function, while the same cells injected in frontal lobes improved cognition (101). Similarly, embryonic motor neurons form connections with mature motor but not visual areas, while embryonic visual neurons connect with mature visual but not motor areas (102). Taken together, these preclinical studies emphasize the importance of timing, location, and dosing, which are rarely standardized in clinical trials. The current literature suggests that it is somewhat premature to focus on these factors, until preclinical studies demonstrate effective delivery of standardized cell-based preparations to desired targets using clinically relevant paradigms and outcome measures.
Other investigators have studied the impact of dosing regimens, including number of cells, dosing frequency, and cell localization (75,102-104). Drobyshevsky et al. (75) elegantly showed early dose-response relationships in efficacy related to human UCB cell number in a rabbit model of $\mathrm{CP}$, using short-term (11 day) outcomes. Infusion of a higher dose of UCB cells mitigated abnormalities in posture, tone, and dystonia, and while half the dose showed limited improvement (75). Moreover, UCB cell tracing with magnetic resonance imaging biomarkers, including Feridex (superparamagnetic iron oxide-labeling) and PCR for human DNA found little penetration of infused cells into the brain in the newborn period, suggesting that short-term benefits were related to paracrine signaling mechanisms as opposed to direct cellular integration or proliferative effects (75). Finally, the timing of cell delivery seems to matter. Cells administered 3 days after injury, but not earlier or later time points, effected repair assessed at 30 days after injury (91). Taken together, these studies emphasize the profound importance of cell type, source, preparation, delivery route, dosing, and timing to efficacy, in addition to confounding factors such as etiology and severity of injury of $\mathrm{CP}$.

\section{TRACKING SHORT- AND LONG-TERM EFFICACY}

Owing to the high burden of disease in people with $\mathrm{CP}$, therapeutic efficacy and reliability of putative treatments is paramount. As indicated above, and by many studies, the number, phenotype, and viability of cells administered to each patient or research subject needs to be characterized and defined. Specifically, the optimal number of cells needed in humans with varying CNS injuries and how the phenotype of the cells to be administered relates to their efficacy in a specific CNS injury is unknown. With autologous stem cells, it is unclear how the health of the patient before the cell harvest contributes to the yield, viability, and phenotype, or the downstream paracrine signaling or beneficial effects. For example, do MSCs or amniotic epithelial cells harvested from a baby with chorioamnionitis and fetal inflammatory response syndrome offer a protective capacity? Similarly, would ideal or "healthy" cells be beneficial in an "unhealthy" neural microenvironment such as exists in preterm infants exposed to chorioamnionitis, term infants with hypoxic-ischemic encephalopathy, or infants with brain trauma? These key preclinical and clinical considerations are addressed in Figures 4 and 5.

Numerous investigators have attempted to address questions related to efficacy and longevity of stem cells in the brain using sophisticated methods of tracking. In addition to labeling cells with preparations of iron nanoparticles like Feridex and Resovist and evaluating with magnetic resonance imaging, magnetic particle spectroscopy may be a quantitative, easy to interpret, noninvasive form of imaging stem cells that could be used as an adjunct to anatomical imaging modalities (105). Specifically, magnetic particle imaging, a novel tomographic technique, may overcome several limitations of traditional magnetic resonance imaging detection 


\section{Cell-based therapies for cerebral palsy $\quad$ ReVieW}

\section{Box $4 \quad$ Gaps in preclinical knowledge}

\section{Technology/techniques}

- How to efficiently and consistently produce cell-based therapies for different disease processes

- What are fast, reliable screens of functional efficacy

Biomarkers of disease process

- How to identify when the local microenvironment is:

o In need of inflammation modulation?

- The most receptive to neural repair?

Types of cells to be used

- Are certain cell-based therapies better for specific etiologies or pathophysiology?

- Do different cell-based therapies protect or support different host cells?

- Do certain cell-based therapies have longer efficacy?

Lessons learned from closely related neurological disorders

- Are disease defined by enzyme loss more amiable to repair than those defined by cell loss?

- Does type of cell-based therapy differ based on etiology or patient age?

Long-term efficacy

- How do cell-based therapies mediate sustained repair?

- What biomarkers of repair correlate with functional improvement?

Preclinical modeling

- Does preclinical injury and efficacy reflect the human condition?

- Are outcome measures sensitive enough for translation to humans?

Figure 4. Preclinical knowledge gaps related to stem cell use in cerebral palsy.

\section{Box 5 Key clinical questions}

1. What is the most efficient production of cell-based therapies for different disease processes?

2. Do different cell-based therapies protect or support different neural cells?

3. Does maturation of the immune system impact cell-based therapy efficacy?

4. Do different cell-based therapies effect longer recovery than others?

5. Do protocols have to be tailored to patient age?

6. Should cell-based therapies for preterm infants differ from term infants?

7. How do we standardize phenotype, viability, and yield for reproducible dosage and delivery of cellbased therapies across patients and centers?

8. How do we optimize cocktail design (agents, timing, dosage, adjunctive therapies) for each neonate?

9. What adverse events should be monitored in all trials regardless of route of administration?

10. Should allogenic cell-based therapies be matched to individual recipients based on age and sex and etiology of brain injury?

11. Are there short-term outcome measures ( 30 days -6 months) for clinical trials that predict long-term (2 years+) benefit?

12. How do we repair the neural network in $\mathrm{CP}$, as opposed to just replacing cells?

13. How do we identify when local CNS microenvironment is need of modulation and most receptive to neural repair?

Figure 5. Key clinical questions related to the use of stem cells for cerebral palsy.

superparamagnetic iron oxide approaches by eliminating hot spots and offering a direct detection and quantification of cells. These techniques may be especially useful to tracking short-term efficacy and confirmation of cell placement in clinical investigations.

With respect to long-term tracking of efficacy and effects on brain circuitry, many approaches have been used (106). Important insights into the functional properties of neural grafts generated from stem cells have been gained from studies at the cellular level and also by assessing their functional impact in animal models of disease (106). Patch-clamping experiments have demonstrated that neurons derived from embryonic stem cells or hiPSCs develop electrophysiological properties, including evoked and spontaneous firing of action potentials and evidence for functional afferent input from host neurons after several weeks (106). Taken together, these data support the view that the development and maturation of implanted neurons generated from human cells are protracted. Sophisticated studies using optogenetic approaches have provided more rigorous analyses and evidence that neurons generated from pluripotent stem cells are not only capable of developing intrinsic electrophysiological properties in vivo but can also establish functional patterns of afferent and efferent connectivity in the host brain $(106,107)$. Indeed, these studies, and those of others, confirm that neuron growth and myelination can take several weeks to months $(106,108)$. The maturation and electrophysiological properties of hiPSC-derived oligodendrocytes have also been described (85). Importantly, those 
investigations revealed that human oligodendrocytes derived from pluripotent stem cells mature with conserved expression of maturation-specific physiological, functional, and channel properties that overlap with rodents, including characteristic developmental switches in glutamate receptor composition (85).

\section{EARLY CLINICAL TRIALS}

Most cell-based early trials for CP have shown that stem cell administration is well tolerated and safe $(22,109-115)$. A recent meta-analysis suggests a moderate short-term positive treatment effect on gross motor outcomes (22). Caution is warranted, however, as standardized long-term outcomes used for other CP intervention trial outcomes have not been used in trials reported to date $(82,110-115)$. No trial thus far has reported primary outcomes that are typical for surgical interventions for CP (116), or for cell-based interventions for pediatric neurodegenerative diseases, such as a $10 \%$ change in GMFM-66 or GMFM-88 at 24 months (117). It is difficult to determine whether effect is of any clinical significance because of the wide age ranges of the participants studied within individual trials (22), and the need to account for developmental curves in assessing motor outcomes $(7,118)$.

\section{CONCLUSIONS}

$\mathrm{CP}$ is a collection of multietiologic disorders unified by early CNS disturbances and motor impairments that presents unique challenges to patients, families and caregivers, and physicians. Given the changing needs of people with $\mathrm{CP}$ throughout their lifespan and the degree of plasticity in the injured CNS, effective, safe evidence-based therapeutic strategies are paramount. Regarding cell-based therapies, rigorous standardization of cell type, source, preparation, delivery route, dosing, and timing have profound implications for efficacy. Confounding factors such as etiology and changing severity of $\mathrm{CP}$ across the lifespan challenge clinical trial design. Just as we expect medications such as antibiotics or chemotherapeutic agents to adhere to stringent guidelines regarding dosing and treatment regimen, cell-based therapies for $\mathrm{CP}$ must also undergo similar rigorous standards. Continued identification of mechanisms of genetic, structural, and functional variables that drive impairment and recovery, and the development of biomarkers of $\mathrm{CP}$, will advance scientific support for use of cell-based therapies. Effective interventions during the acute, subacute, and repair phases following injury could possibly mitigate or lessen the long-term disability in person with CP. Increased rigor of both preclinical and clinical efforts is essential to move the field forward and improve precision diagnosis and treatment for each individual with $\mathrm{CP}$.

\section{ACKNOWLEDGMENTS}

We are grateful to Tracylyn Yellowhair for helpful assistance with the manuscript.
Disclosure: The authors declare they have no financial relationships and nothing to disclose.

\section{REFERENCES}

1. Rosenbaum P, Paneth N, Leviton A, et al. A report: the definition and classification of cerebral palsy April 2006. Dev Med Child Neurol Suppl 2007;109:8-14.

2. Toyokawa S, Maeda E, Kobayashi Y. Estimation of the number of children with cerebral palsy using nationwide health insurance claims data in Japan. Dev Med Child Neurol 2017;59:317-21.

3. Durkin MS, Benedict RE, Christensen D, et al. Prevalence of cerebral palsy among 8-year-old children in 2010 and preliminary evidence of trends in its relationship to low birthweight. Paediatr Perinat Epidemiol 2016;30:496-510.

4. Hollung SJ, Vik T, Wiik R, et al. Completeness and correctness of cerebral palsy diagnoses in two health registers: implications for estimating prevalence. Dev Med Child Neurol 2017;59:402-6.

5. Alriksson-Schmidt A, Nordmark E, Czuba T, et al. Stability of the Gross Motor Function Classification System in children and adolescents with cerebral palsy: a retrospective cohort registry study. Dev Med Child Neurol 2017;59:641-6.

6. Palisano R, Rosenbaum P, Walter S, et al. Development and reliability of a system to classify gross motor function in children with cerebral palsy. Dev Med Child Neurol 1997;39:214-23.

7. Rosenbaum PL, Walter SD, Hanna SE, et al. Prognosis for gross motor function in cerebral palsy: creation of motor development curves. JAMA 2002;288:1357-63.

8. Stavsky M, Mor O, Mastrolia SA, et al. Cerebral palsy-trends in epidemiology and recent development in prenatal mechanisms of disease, treatment, and prevention. Front Pediatr 2017;5:21.

9. Novak I, Hines M, Goldsmith S, et al. Clinical prognostic messages from a systematic review on cerebral palsy. Pediatrics 2012;130:e1285-312.

10. Monbaliu E, De La Pena MG, Ortibus E, et al. Functional outcomes in children and young people with dyskinetic cerebral palsy. Dev Med Child Neurol 2017;59:634-40.

11. Anblagan D, Pataky R, Evans MJ, et al. Association between preterm brain injury and exposure to chorioamnionitis during fetal life. Sci Rep 2016;6:37932.

12. Ahlin K, Jacobsson B, Nilsson S, et al. Antecedents and neuroimaging patterns in cerebral palsy with epilepsy and cognitive impairment: a population-based study in children born at term. Acta Obstet Gynecol Scand 2017;96:828-36.

13. Nelson KB, Blair E. Prenatal factors in singletons with cerebral palsy born at or near term. N Engl J Med 2015;373:946-53.

14. Leviton A, Fichorova RN, O'Shea TM, et al. Two-hit model of brain damage in the very preterm newborn: small for gestational age and postnatal systemic inflammation. Pediatr Res 2013;73:362-70.

15. Korzeniewski SJ, Romero R, Cortez J, et al. A "multi-hit" model of neonatal white matter injury: cumulative contributions of chronic placental inflammation, acute fetal inflammation and postnatal inflammatory events. J Perinat Med 2014;42:731-43.

16. Lungu C, Hirtz D, Damiano D, et al. Report of a workshop on research gaps in the treatment of cerebral palsy. Neurology 2016;87: 1293-8.

17. Novak I, McIntyre S, Morgan C, et al. A systematic review of interventions for children with cerebral palsy: state of the evidence. Dev Med Child Neurol 2013;55:885-910.

18. Masgrau R, Guaza C, Ransohoff RM, et al. Should we stop saying 'glia' and 'neuroinflammation'? Trends Mol Med 2017;23:486-500.

19. Muraro PA, Pasquini M, Atkins HL, et al. Long-term outcomes after autologous hematopoietic stem cell transplantation for multiple sclerosis. JAMA Neurol 2017;74:459-69.

20. Goldman SA, Kuypers NJ. How to make an oligodendrocyte. Development 2015;142:3983-95.

21. Carroll JE, Mays RW. Update on stem cell therapy for cerebral palsy. Expert Opin Biol Ther 2011;11:463-71. 
22. Novak I, Walker K, Hunt RW, et al. Concise review: stem cell interventions for people with cerebral palsy: systematic review with meta-analysis. Stem Cells Transl Med 2016;5:1014-25.

23. Ahn SY, Chang YS, Park WS. Stem cells for neonatal brain disorders. Neonatology 2016;109:377-83.

24. Chang YS, Ahn SY, Sung S, et al. Stem cell therapy for neonatal disorders: prospects and challenges. Yonsei Med J 2017;58:266-71.

25. Kiasatdolatabadi A, Lotfibakhshaiesh N, Yazdankhah M, et al. The role of stem cells in the treatment of cerebral palsy: a review. Mol Neurobiol 2016;54:4963-72.

26. Bennet L, Tan S, Van den Heuij L, et al. Cell therapy for neonatal hypoxia-ischemia and cerebral palsy. Ann Neurol 2012;71:589-600.

27. Daley GQ. Polar extremes in the clinical use of stem cells. N Engl J Med 2017;376:1075-7.

28. Kimmelman J, Hyun I, Benvenisty N, et al. Policy: global standards for stem-cell research. Nature 2016;533:311-3.

29. Caulfield T, Sipp D, Murry CE, et al. Scientific community. Confronting stem cell hype. Science 2016;352:776-7.

30. Daley GQ, Hyun I, Apperley JF, et al. Setting global standards for stem cell research and clinical translation: the 2016 ISSCR Guidelines. Stem Cell Rep 2016;6:787-97.

31. Cote DJ, Bredenoord AL, Smith TR, et al. Ethical clinical translation of stem cell interventions for neurologic disease. Neurology 2017;88:322-8.

32. Sharpe K, Di Pietro N, Jacob KJ, et al. A dichotomy of informationseeking and information-trusting: stem cell interventions and children with neurodevelopmental disorders. Stem Cell Rev 2016;12:438-47.

33. Anderson AJ, Piltti KM, Hooshmand MJ, et al. Preclinical efficacy failure of human CNS-derived stem cells for use in the pathway study of cervical spinal cord injury. Stem Cell Rep 2017;8:249-63.

34. Coq JO, Delcour M, Massicotte VS, et al. Prenatal ischemia deteriorates white matter, brain organization, and function: implications for prematurity and cerebral palsy. Dev Med Child Neurol 2016;58 (Suppl 4): 7-11.

35. Delcour M, Russier M, Amin M, et al. Impact of prenatal ischemia on behavior, cognitive abilities and neuroanatomy in adult rats with white matter damage. Beh Brain Res 2012;232:233-44.

36. Robinson S, Petelenz K, Li Q, et al. Developmental changes induced by prenatal hypoxia-ischemia insult in rats models human perinatal brain injury. Neurobiol Dis 2005;18:568-81.

37. Jantzie LL, Corbett CJ, Berglass J, et al. Complex pattern of interaction between in utero hypoxia-ischemia and intra-amniotic inflammation disrupts brain development and motor function. J Neuroinflamm 2014;11:131

38. Jantzie LL, Robinson S. Preclinical models of encephalopathy of prematurity. Dev Neurosci 2015;37:277-88.

39. McCauley S, Wilde E, Anderson V, et al. Recommendations for use of the common outcome measures in pediatric traumatic brain injury research. J Neurotrauma 2012;29:678-705.

40. Thomason ME, Scheinost D, Manning JH, et al. Weak functional connectivity in the human fetal brain prior to preterm birth. Sci Rep 2017;7:39286.

41. Leviton A, Allred EN, Fichorova RN, et al. Systemic inflammation on postnatal days 21 and 28 and indicators of brain dysfunction 2years later among children born before the 28th week of gestation. Early Hum Dev 2016;93:25-32.

42. Wu Y. Systematic review of chorioamnionitis and cerebral palsy. Ment Retard Dev Disabil Res Rev 2002;8:25-9.

43. Paton MCB, McDonald CA, Allison BJ, et al. Perinatal brain injury as a consequence of preterm birth and intrauterine inflammation: designing targeted stem cell therapies. Front Neurosci 2017;11:200.

44. Shatrov JG, Birch SC, Lam LT, et al. Chorioamnionitis and cerebral palsy: a meta-analysis. Obstet Gynecol 2010;116 (Part 1)); 387-92.

45. Kuypers E, Ophelders D, Jellema RK, et al. White matter injury following fetal inflammatory response syndrome induced by chorioamnionitis and fetal sepsis: lessons from experimental ovine models. Early Hum Dev 2012;88:931-6.
46. Synnes A, Anderson PJ, Grunau RE, et al. Predicting severe motor impairment in preterm children at age 5 years. Arch Dis Child 2015;100: $748-53$.

47. Kiray H, Lindsay SL, Hosseinzadeh S, et al. The multifaceted role of astrocytes in regulating myelination. Exp Neurol 2016;283 (Part B)); $541-9$.

48. Robinson S, Li Q, DeChant A, et al. Neonatal loss of gamma amino butyric acid pathway expression after human perinatal brain injury. J Neurosurg 2006;104:396-408.

49. Pogledic I, Kostovic I, Fallet-Bianco C, et al. Involvement of the subplate zone in preterm infants with periventricular white matter injury. Brain Pathol 2014;24:128-41.

50. Kinney HC, Haynes RL, Xu G, et al. Neuron deficit in the white matter and subplate in periventricular leukomalacia. Ann Neurol 2012;71: 397-406.

51. Volpe J. Brain injury in premature infants: a complex amalgam of destructive and developmental disturbances. Lancet Neurol 2009;8: $110-24$.

52. Brandao JA, Romcy-Pereira RN. Interplay of environmental signals and progenitor diversity on fate specification of cortical GABAergic neurons. Front Cell Neurosci 2015;9:149.

53. Hung CC, Lin $\mathrm{CH}$, Chang $\mathrm{H}$, et al. Astrocytic GAP43 induced by the TLR4/NF-kappaB/STAT3 axis attenuates astrogliosis-mediated microglial activation and neurotoxicity. J Neurosci 2016;36:2027-43.

54. Matcovitch-Natan O, Winter DR, Giladi A, et al. Microglia development follows a stepwise program to regulate brain homeostasis. Science 2016;353:aad8670.

55. Grabert K, Michoel T, Karavolos MH, et al. Microglial brain regiondependent diversity and selective regional sensitivities to aging. Nat Neurosci 2016;19:504-16.

56. Mosser CA, Baptista S, Arnoux I, et al. Microglia in CNS development: shaping the brain for the future. Prog Neurobiol 2017;149-150:1-20.

57. Jantzie LL, Corbett CJ, Firl DJ, et al. Postnatal erythropoietin mitigates impaired cerebral cortical development following subplate loss from prenatal hypoxia-ischemia. Cereb Cortex 2014;25:2683-95.

58. Jantzie LL, Talos DM, Jackson MC, et al. Developmental expression of $\mathrm{N}$-methyl-D-aspartate (NMDA) receptor subunits in human white and gray matter: potential mechanism of increased vulnerability in the immature brain. Cereb Cortex 2013;25:482-95.

59. Robinson S, Mikolaenko I, Thompson I, et al. Loss of cation-chloride cotransporter expression in preterm infants with white matter lesions: implications for the pathogenesis of epilepsy. J Neuropathol Exp Neurol 2010;69:565-72.

60. Lin CY, Chang YC, Wang ST, et al. Altered inflammatory responses in preterm children with cerebral palsy. Ann Neurol 2010;68:204-12.

61. Rajantie I, Ilmonen M, Alminaite A, et al. Adult bone marrow-derived cells recruited during angiogenesis comprise precursors for periendothelial vascular mural cells. Blood 2004;104:2084-6.

62. Reyes M, Dudek A, Jahagirdar B, et al. Origin of endothelial progenitors in human postnatal bone marrow. J Clin Invest 2002;109: $337-46$.

63. Riess P, Zhang C, Saatman KE, et al. Transplanted neural stem cells survive, differentiate, and improve neurological motor function after experimental traumatic brain injury. J Clin Invest 2002;51: 1043-52; discussion 1052-1054.

64. Sanberg PR, Eve DJ, Metcalf C, et al. Advantages and challenges of alternative sources of adult-derived stem cells for brain repair in stroke. Prog Brain Res 2012;201:99-117.

65. Liu S, de Castro LF, Jin P, et al. Manufacturing differences affect human bone marrow stromal cell characteristics and function: comparison of production methods and products from multiple centers. Sci Rep 2017;7: 46731

66. McKenna D. Challenges in the cGMP manufacturing of MSCs for multicenter academia trials. Transfusion 2016;56:18S-19SS. 
67. Pamies D, Bal-Price A, Simeonov A, et al. Good cell culture practice for stem cells and stem-cell-derived models. ALTEX 2017;34: 95-132.

68. Erices A, Conget P, Minguell JJ. Mesenchymal progenitor cells in human umbilical cord blood. Br J Haematol 2000;109:235-42.

69. Nieda M, Nicol A, Denning-Kendall P, et al. Endothelial cell precursors are normal components of human umbilical cord blood. Br J Haematol 1997;98:775-7.

70. Park DH, Lee JH, Borlongan CV, et al. Transplantation of umbilical cord blood stem cells for treating spinal cord injury. Stem Cell Rev 2011;7: 181-94.

71. Park DH, Borlongan CV, Willing AE, et al. Human umbilical cord blood cell grafts for brain ischemia. Cell Transplant 2009;18:985-98.

72. Wen SH, Zhao WL, Lin PY, et al. Associations among birth weight, placental weight, gestational period and product quality indicators of umbilical cord blood units. Transfus Apher Sci 2012;46:39-45.

73. Li J, McDonald CA, Fahey MC, et al. Could cord blood cell therapy reduce preterm brain injury? Front Neurol 2014;5:200.

74. Mazzoccoli G, Miscio G, Fontana A, et al. Time related variations in stem cell harvesting of umbilical cord blood. Sci Rep 2016;6:21404.

75. Drobyshevsky A, Cotten CM, Shi Z, et al. Human umbilical cord blood cells ameliorate motor deficits in rabbits in a cerebral palsy model. Dev Neurosci 2015;37:349-62.

76. Hass R, Kasper C, Bohm S, et al. Different populations and sources of human mesenchymal stem cells (MSC): a comparison of adult and neonatal tissue-derived MSC. Cell Commun Signal 2011;9:12.

77. Jacobs SA, Roobrouck VD, Verfaillie CM, et al. Immunological characteristics of human mesenchymal stem cells and multipotent adult progenitor cells. Immunol Cell Biol 2013;91:32-9.

78. Kern S, Eichler H, Stoeve J, et al. Comparative analysis of mesenchymal stem cells from bone marrow, umbilical cord blood, or adipose tissue. Stem Cells 2006;24:1294-301.

79. Amable PR, Teixeira MV, Carias RB, et al. Protein synthesis and secretion in human mesenchymal cells derived from bone marrow, adipose tissue and Wharton's jelly. Stem Cell Res Ther 2014;5:53.

80. Gonzales-Portillo GS, Reyes S, Aguirre D, et al. Stem cell therapy for neonatal hypoxic-ischemic encephalopathy. Front Neurol 2014;5: 147.

81. Bae SH, Kong TH, Lee HS, et al. Long-lasting paracrine effects of human cord blood cells on damaged neocortex in an animal model of cerebral palsy. Cell Transplant 2012;21:2497-515.

82. Min K, Song J, Kang JY, et al. Umbilical cord blood therapy potentiated with erythropoietin for children with cerebral palsy: a double-blind, randomized, placebo-controlled trial. Stem Cells 2013;31:581-91.

83. Bae SH, Lee HS, Kang MS, et al. The levels of pro-inflammatory factors are significantly decreased in cerebral palsy patients following an allogeneic umbilical cord blood cell transplant. Int J Stem Cells 2012;5: $31-8$.

84. Wang S, Bates J, Li X, et al. Human iPSC-derived oligodendrocyte progenitor cells can myelinate and rescue a mouse model of congenital hypomyelination. Cell Stem Cell 2013;12:252-64.

85. Livesey MR, Magnani D, Cleary EM, et al. Maturation and electrophysiological properties of human pluripotent stem cell-derived oligodendrocytes. Stem Cells 2016;34:1040-53.

86. Fan HC, Ho LI, Chi CS, et al. Current proceedings of cerebral palsy. Cell Transplant 2015;24:471-85.

87. Yawno T, Schuilwerve J, Moss TJ, et al. Human amnion epithelial cells reduce fetal brain injury in response to intrauterine inflammation. Dev Neurosci 2013;35:272-82.

88. Donega V, Nijboer CH, van Tilborg G, et al. Intranasally administered mesenchymal stem cells promote a regenerative niche for repair of neonatal ischemic brain injury. Exp Neurol 2014;261:53-64.

89. Berger R, Soder S. Neuroprotection in preterm infants. Biomed Res Int 2015;2015:257139.

90. Chen S, Lewallen M, Xie T. Adhesion in the stem cell niche: biological roles and regulation. Development 2013;140:255-65.
91. Rosenblum S, Wang N, Smith TN, et al. Timing of intra-arterial neural stem cell transplantation after hypoxia-ischemia influences cell engraftment, survival, and differentiation. Stroke 2012;43:1624-31.

92. Kim DK, Nishida H, An SY, et al. Chromatographically isolated CD63 +CD81+ extracellular vesicles from mesenchymal stromal cells rescue cognitive impairments after TBI. Proc Natl Acad Sci USA 2016;113:170-5.

93. Drommelschmidt K, Serdar M, Bendix I, et al. Mesenchymal stem cellderived extracellular vesicles ameliorate inflammation-induced preterm brain injury. Brain Behav Immun 2017;60:220-32.

94. Sweda R, Phillips AW, Marx J, et al. Glial-restricted precursors protect neonatal brain slices from hypoxic-ischemic cell death without direct tissue contact. Stem Cells Dev 2016;25:975-85.

95. Teixeira FG, Carvalho MM, Sousa N, et al. Mesenchymal stem cells secretome: a new paradigm for central nervous system regeneration? Cell Mol Life Sci 2013;70:3871-82.

96. Pires AO, Mendes-Pinheiro B, Teixeira FG, et al. Unveiling the differences of secretome of human bone marrow mesenchymal stem cells, adipose tissue-derived stem cells, and human umbilical cord perivascular cells: a proteomic analysis. Stem Cells Dev 2016;25:1073-83.

97. Salgado AJ, Sousa JC, Costa BM, et al. Mesenchymal stem cells secretome as a modulator of the neurogenic niche: basic insights and therapeutic opportunities. Front Cell Neurosci 2015;9:249.

98. Teixeira FG, Panchalingam KM, Anjo SI, et al. Do hypoxia/normoxia culturing conditions change the neuroregulatory profile of Wharton Jelly mesenchymal stem cell secretome? Stem Cell Res Ther 2015;6:133.

99. Shapira I, Fainstein N, Tsirlin M, et al. Placental stromal cell therapy for experimental autoimmune encephalomyelitis: the role of route of cell delivery. Stem Cells Transl Med 2017;6:1286-94.

100. Shroff G, Gupta A, Barthakur JK. Therapeutic potential of human embryonic stem cell transplantation in patients with cerebral palsy. J Transl Med 2014;12:318.

101. Piao J, Major T, Auyeung G, et al. Human embryonic stem cell-derived oligodendrocyte progenitors remyelinate the brain and rescue behavioral deficits following radiation. Cell Stem Cell 2015;16:198-210.

102. Michelsen KA, Acosta-Verdugo S, Benoit-Marand M, et al. Area-specific reestablishment of damaged circuits in the adult cerebral cortex by cortical neurons derived from mouse embryonic stem cells. Neuron 2015;85:982-97.

103. Cameron SH, Alwakeel AJ, Goddard L, et al. Delayed post-treatment with bone marrow-derived mesenchymal stem cells is neurorestorative of striatal medium-spiny projection neurons and improves motor function after neonatal rat hypoxia-ischemia. Mol Cell Neurosci 2015;68:56-72.

104. van Velthoven CT, Sheldon RA, Kavelaars A, et al. Mesenchymal stem cell transplantation attenuates brain injury after neonatal stroke. Stroke 2013;44:1426-32.

105. Bulte JW, Walczak P, Janowski M, et al. Quantitative "hot spot" imaging of transplanted stem cells using superparamagnetic tracers and magnetic particle imaging (MPI). Tomography 2015;1:91-7.

106. Thompson LH, Bjorklund A. Reconstruction of brain circuitry by neural transplants generated from pluripotent stem cells. Neurobiol Dis 2015;79:28-40.

107. Steinbeck JA, Choi SJ, Mrejeru A, et al. Optogenetics enables functional analysis of human embryonic stem cell-derived grafts in a Parkinson's disease model. Nat Biotechnol 2015;33:204-9.

108. Espuny-Camacho I, Michelsen KA, Gall D, et al. Pyramidal neurons derived from human pluripotent stem cells integrate efficiently into mouse brain circuits in vivo. Neuron 2013;77:440-56.

109. Feng M, Lu A, Gao H, et al. Safety of allogeneic umbilical cord blood stem cells therapy in patients with severe cerebral palsy: A Retrospective Study. Stem Cells Int 2015;2015:325652.

110. Rah WJ, Lee YH, Moon JH, et al. Neuroregenerative potential of intravenous G-CSF and autologous peripheral blood stem cells in children with cerebral palsy: a randomized, double-blind, crossover study. J Transl Med 2017;15:16. 
111. Liu X, Fu X, Dai G, et al. Comparative analysis of curative effect of bone marrow mesenchymal stem cell and bone marrow mononuclear cell transplantation for spastic cerebral palsy. J Transl Med 2017;15:48.

112. Chen $\mathrm{L}$, Huang $\mathrm{H}, \mathrm{Xi} \mathrm{H}$, et al. Intracranial transplant of olfactory ensheathing cells in children and adolescents with cerebral palsy: a randomized controlled clinical trial. Cell Transplant 2010;19:185-91.

113. Kang M, Min K, Jang J, et al. Involvement of immune responses in the efficacy of cord blood cell therapy for cerebral palsy. Stem Cells Dev 2015;24:2259-68.

114. Luan Z, Liu W, Qu S, et al. Effects of neural progenitor cell transplantation in children with severe cerebral palsy. Cell Transplant 2012;21 (Suppl 1)); S91-8.
115. Chen G, Wang Y, Xu Z, et al. Neural stem cell-like cells derived from autologous bone mesenchymal stem cells for the treatment of patients with cerebral palsy. J Transl Med 2013;11:21.

116. Dudley RW, Parolin M, Gagnon B, et al. Long-term functional benefits of selective dorsal rhizotomy for spastic cerebral palsy. J Neurosurg Pediatr 2013;12:142-50.

117. Sessa M, Lorioli L, Fumagalli F, et al. Lentiviral haemopoietic stem-cell gene therapy in early-onset metachromatic leukodystrophy: an ad-hoc analysis of a non-randomised, open-label, phase 1/2 trial. Lancet 2016;388:476-87.

118. Hanna SE, Bartlett DJ, Rivard LM, et al. Reference curves for the Gross Motor Function Measure: percentiles for clinical description and tracking over time among children with cerebral palsy. Phys Ther 2008;88:596-607. 